\title{
Contribution à l'élevage de l'escargot Petit-gris : Helix aspersa Müller (Mollusque gastéropode pulmoné stylommatophore). II. - Evolution de la population juvénile de l'éclosion à l'âge de 12 semaines, en bâtiment et en conditions d'élevage contrôlées
}

\author{
J. DAGUZAN \\ avec la collaboration technique de J.C. Bonnet*, Yvette Perrin*, E. Perrin* \\ et H. ROUET * \\ Laboratoire de Zoologie générale et d'Ecophysiologie, \\ U.E.R. des Sciences de la Vie et de l'Environnement, \\ Université de Rennes I \\ Avenue du Général-Leclerc, $F 35042$ Rennes Cedex \\ * I.N.R.A., Centre hélicicole de la Station du Magneraud \\ Saint-Pierre-d'A milly, B.P. 52, F 17700 Surgères
}

\section{Résumé}

Les principaux résultats concernant la croissance des escargots Petits-gris (Helix aspersa Müller), de leur éclosion jusqu'à l'âge de 12 semaines, en élevage effectué en bâtiment hors sol et en conditions thermo-hygrométriques contrôlées, sont présentés dans ce mémoire.

Cette phase de l'héliciculture relative au stade juvénile I (grand diamètre de la coquille D compris entre 3 et $22 \mathrm{~mm}$ ) comporte tout d'abord la croissance des " jeunes 1 » (nursery $1^{\text {er }}$ âge), puis celle des "jeunes $2 \gg$ (nursery $2^{\text {" }}$ âge). Certains tris des individus selon leur taille permettent de diminuer l'hétérogénéité des populations et le rattrapage du retard de croissance des individus Ics plus faibles. Enfin, il semble que la charge biotique ait une influence très importante sur la croissance des individus.

\section{Introduction}

Chez les Mollusques Gastéropodes Pulmonés terrestres, la croissance peut être de deux formes, l'une de type continu que l'on observe surtout chez les limaces arionidae (Abeloos, 1944; Stern, 1968; Chevallier, 1969), et l'autre de type discontinue, c'est-à-dire présentant des arrêts de croissance lorsque les conditions deviennent défavorables, et que l'on enregistre chez divers escargots tels que Cepaea nemoralis L. (LAmotTe, 1951 ; Wolda, 1963, 1972, 1973 ; Williamson, 1976), Theba pisana (Müller) (Lazaridou-Dimitriadou, 1978), Helix pomatia L. (Pollard, 1973,

Ce travail a été réalisé dans le cadre d'une action concertée I.T.A.V.I., I.N.R.A., Université de Rennes I, et grâce à un financement de l'E.P.R. de la Région Poitou-Charentes. 
1975 ; Pollard, Cooke \& Welch, 1977) et Helix aspersa Müller (Potts, 1972 ; Charrier \& Daguzan, 1979 ; Charrier, 1980).

De plus, chez les Gastéropodes Pulmonés, nombreux sont les facteurs abiotiques (climatiques, nutritionnels) ou biotiques (âge, densité, génétique) qui conditionnent la croissance, aussi bien au point de vue de sa vitesse qu'au niveau de la taille limite. Ainsi, chez Cepaea nemoralis L., l'augmentation de la densité des individus favorise, dans un premier temps, la locomotion et l'ingestion de nourriture, mais provoque par la suite une sécrétion importante de mucus qui inhiberait à son tour la locomotion et la consommation alimentaire, donc indirectement la croissance des individus (OOSTERHOFF, 1977). De même la température et la photo-période ont un rôle important sur l'accroissement de volume de la coquille de l'escargot Petit-gris (Helix aspersa Müll.) (Herzberg \& HerzberG, 1960). Le facteur "groupement 》 (agrégat) des individus a une action prépondérante sur la croissance, la production et la mortalité des limaces et des escargots. Ce phénomène est mis en évidence chez les Arionidae (CHEvalLIER, 1974), Cepaea nemoralis (OosterhofF, 1977), Theba pisana (LAZARIdou-DimiTriadou \& Daguzan, 1981) et Helix aspersa (Charrier, 1980).

Enfin, il existe une grande variabilité de la vitesse de croissance entre les diverses populations et même au niveau des individus issus d'une même ponte, aussi bien chez Cepaea nemoralis (Wolda. 1971 ; WILliamson, 1976 ; Oosterhoff, 1977) que chez Helix pomatia (Pollard, Cooke \& Welch, 1977). Cela est dû probablement d'une part à des facteurs génétiques et, d'autre part à l'environnement.

En ce qui concerne l'escargot Petit-gris (Helix aspersa), on sait que sa croissance comporte trois phases correspondant à trois états de maturité sexuelle (CHARRIER \& Daguzan, 1979) :

- un stade juvénile $I(3,0 \leqslant \mathrm{D}<22,0 \mathrm{~mm})$ '1) caractérisé par un appareil génital très peu différencié ;

- un stade juvénile II $(22,0 \leqslant \mathrm{D}<27,0 \mathrm{~mm})$ durant lequel s'effectue la différenciation de certains éléments de l’appareil génital (pénis, glande à albumen, glandes multifides) ;

- un stade adulte $(\mathrm{D} \geqslant 27,0 \mathrm{~mm})$ présentant un appareil génital bien différencié et fonctionnel, avec un dard à 4 arêtes dans la poche à dard.

Après avoir exposé antérieurement les résultats concernant la reproduction et l'éclosion des jeunes individus d'Helix aspersa, en bâtiment et en conditions thermohygrométriques contrôlées (DAGUZAN, 1981), il nous a paru intéressant de présenter les principaux résultats relatifs à la seconde phase de l'élevage, réalisée dans le bâtiment «nursery » et concernant la croissance des jeunes escargots depuis leur naissance jusqu'à l'âge de 12 semaines (stade juvénile I). Cette phase de l'héliciculture comporte tout d'abord la croissance des «jeunes $1 \gg$ (nursery $1^{\text {er }}$ âge) de l'éclosion à 6 semaines, puis celle des "jeunes $2 \gg$ (nursery 2 " âge) de l'âge de 6 semaines à celui de 12 semaines.

(1) La mesure D correspond à la valeur du grand diamètre de la coquille. 


\section{Techniques expérimentales et matériel utilisé}

\section{Caractéristiques du bâtiment «écloserie-nursery» et des enceintes d'élevage type «nursery»}

a) Aménagement et climatisation du bâtiment

Dans ce bâtiment d'une surface de $109 \mathrm{~m}^{2}$, se dressent 10 portiques métalliques constitués de «Dexion » comportant chacun 5 étagères permettant de disposer 10 enceintes d'élevage par niveau, soit 50 par portique.

La température, l'humidité relative et la lumière sont contrôlées (tabl. 1).

\section{TABLEAU 1}

Caractéristiques climatiques du bâtiment «écloserie-nursery».

Climatic characteristics of the «hatching-nursery 》 building.

- Température ambiante : $20 \pm 2{ }^{\circ} \mathrm{C}$

- Humidité relative :

Jour : 65 à $75 \%$

Nuit : 85 à $95 \%$

- Photopériode artificielle : «lumière du jour»:

Jour : 8 heures $(8 \mathrm{~h} 30-16$ h 30$)$

Nuit : 16 heures $(16$ h $30-8$ h 30$)$

b) Enceinte d'élevage type «nursery»

- Type «nursery $1^{\text {er }}$ âge»

Chaque enceinte d'élevage est constituée d'une boîte en bois contreplaqué $(39,5 \times 24,0 \times 12,5 \mathrm{~cm})$ représentant une surface au sol de $0,081 \mathrm{~m}^{2}$ et un volume disponible de $0,010 \mathrm{~m}^{3}$ (volume uniquement utilisable, c'est-à-dire diminué du volume correspondant aux abris et structures intérieures de l'enceinte) (fig. 1). Le couvercle de la boîte est constitué par un cadre de bois grillagé. Enfin, le plancher de l'enceinte est garni d'une couche de mousse plastique imbibée d'eau.

\section{- Type «nursery $2^{\circ}$ âge»}

Il s'agit d'une boîte de polystyrène $(50,5 \times 35,5 \times 17,5 \mathrm{~cm})$ ayant une surface au sol de $0,138 \mathrm{~m}^{2}$ et un volume disponible de $0,019 \mathrm{~m}^{3}$ (fig. 2). Le couvercle de l'enceinte et deux des côtés présentent une ouverture garnie d'un grillage à maille carrée afin que l'intérieur soit bien aéré et éclairé. De plus, on augmente la surface 
de «collage» des escargots en ajoutant 2 grands abris constitués de morceaux de polystyrène. Enfin, le plancher de polystyrène de l'enceinte est recouvert d'une toile plastique facilitant le nettoyage réalisé une fois par semaine.

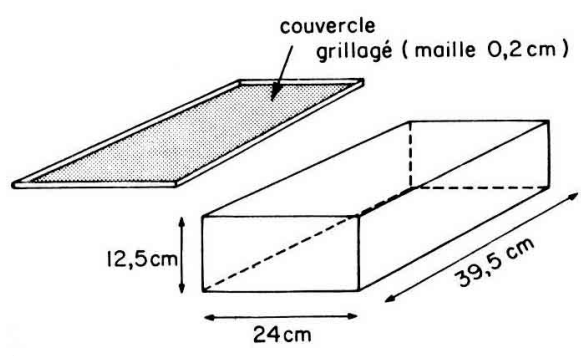

FIG. 1

Schéma d'une enceinte d'élevage type «nursery $1^{\text {er }}$ âge».

Scheme of a rearing-box, type "nursery $1 》$.

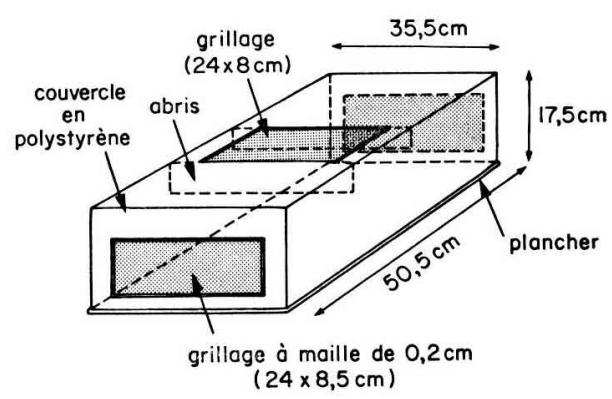

FIG. 2

Schéma d'une enceinte d'élevage type «nursery $2^{\circ}$ âge».

Scheme of a rearing-box, type "nursery $2 »$.

\section{Alimentation}

La nourriture fournie aux escargots est de l'aliment composé «Sanders 》, sous forme de poudre, placé dans de petits récipients plastiques. De plus, on y ajoute 10 p. 100 de maerl lavé à l'eau douce et pulvérisé.

Enfin, des abreuvoirs remplis toujours d'eau permettent aux animaux de se désaltérer fréquemment.

\section{Charge biotique des enceintes d'élevage type «nursery»}

a) Type «nursery $1^{\text {er }}$ age»

Dans chaque enceinte, on place une biomasse initiale de $50 \mathrm{~g}$ d'escargots âgés de 1 jour, ce qui correspond à environ 2000 individus, cette valeur ayant été déterminée, à la suite de recherches préalables (tabl. 2). Comme nous l'avons déjà signalé (Daguzan, 1981), il est préférable de rapporter la charge biotique au volume disponible, plutôt qu'à la surface totale de collage, non pas pour définir le volume d'air disponible, mais pour quantifier l'espace vital des individus, notion fondamentale pour des Gastéropodes Pulmonés.

b) Type «nursery $2^{\circ}$ âge»

La charge biotique est de $80 \mathrm{~g}$ d'escargots par enceinte. La densité varie selon la taille des individus placés dans les boîtes; elle oscille entre 1450 individus par $\mathrm{m}^{2}$ et 7250 individus par $\mathrm{m}^{2}$ au sol (tabl. 3). 


\section{Tableau 2}

Caractéristiques des enceintes d'élevage «nursery $1^{\text {er }}$ âge».

Characteristics of $"$ nursery $1 \gg$ rearing-boxes.

- Effectif : 2000 individus

- Biomasse : $50,45 \pm 0,40 \mathrm{~g} \mathrm{(*)}$ (C.V. $=0,8 \%$ )

- Poids moyen des escargots : $28,0 \pm 8,0 \mathrm{mg}$ (C.V. $=29 \%$ )

- Densité au $\mathrm{m}^{2}$ de sol : 24700 individus

- Charge biotique $/ \mathrm{m}^{2}$ sol : $630 \mathrm{~g}$

- Charge biotique $/ \mathrm{m}^{3}$ disponible : $5 \mathrm{~kg}$

(*) Moyenne \pm écart-type.

C.V. : coefficient de variation.

Tableau 3

Caractéristiques des enceintes d'élevage «nursery $2^{\circ}$ âge 》.

Characteristics of «nursery $2 》$ rearing-boxes.

\begin{tabular}{|c|c|c|}
\hline Paramètres & $\begin{array}{c}\text { Enceintes } \\
\text { avec individus } \\
\text { ayant } \mathrm{D}<6 \mathrm{~mm}\end{array}$ & $\begin{array}{c}\text { Enceintes } \\
\text { avec individus } \\
\text { ayant } D \geqslant 6 \mathrm{~mm}\end{array}$ \\
\hline Effectif moyen .... & 1000 & 200 \\
\hline Biomasse moyenne & $\begin{array}{l}80,23 \pm 0,16 \mathrm{~g} \\
(\mathrm{C} . \mathrm{V} .=0,2 \%)\end{array}$ & $\begin{array}{l}80,28 \pm 0,16 \mathrm{~g} \\
(\mathrm{C} . \mathrm{V} .=0,2 \%)\end{array}$ \\
\hline Poids moyen des escargots $\ldots$. & $\begin{array}{l}82,8 \pm 42,4 \mathrm{mg} \\
(\mathrm{C} . \mathrm{V} .=51 \%)\end{array}$ & $\begin{array}{c}360,6 \pm 203,0 \mathrm{mg} \\
(\mathrm{C} . \mathrm{V} .=56 \%)\end{array}$ \\
\hline Densité au $\mathrm{m}^{2}$ de sol $\ldots$ & 7250 individus & 1450 individus \\
\hline Charge biotique $/ \mathrm{m}^{2}$ sol $\ldots$. & $580 \mathrm{~g}$ & $580 \mathrm{~g}$ \\
\hline Charge biotique $/ \mathrm{m}^{3}$ disponible & $4,2 \mathrm{~kg}$ & $4,2 \mathrm{~kg}$ \\
\hline
\end{tabular}

Moyenne \pm écart-type.

4. Tri

L'existence d'une grande hétérogénéité de tailles ou de poids a pu être mise en évidence chez plusieurs Gastéropodes Pulmonés, en particulier chez Cepaea nemoralis (OOSTERHOFF, 1977), Theba pisana (LAZARIdou-Dimitriadou \& DAgUzAN, 1981), Helix aspersa (Charrier, 1980) et Elonaquimperiana (de Ferussac) (Daguzan, 1982). De plus, de nombreuses expériences réalisées au laboratoire ont montré que des 
populations de jeunes ecargots Petit-gris, de tailles initiales sensiblement identiques, élevées dans diverses enceintes, présentaient très rapidement une forte hétérogénéité de tailles donc de poids, puisqu'il existe une très bonne corrélation entre ces deux paramètres (Charrier \& Daguzan, 1979). Par la suite, seuls les individus les plus gros continuent de grossir tandis que les petits dépérissent et meurent.

Ainsi, on suit tout particulièrement l'évolution du coefficient de variation (C.V.) du poids des escargots élevés dans deux enceintes «nursery $1^{\text {er }}$ âge » (soit 4000 individus). Dès que ce coefficient atteint une valeur trop élevée (C.V. $>80$ p. 100), on effectue un premier tri des individus, afin d'homogénéiser la distribution de tailles des escargots. Cette opération a lieu ainsi à lâge de 6 semaines et marque la fin de la phase «nursery $1^{\text {er }}$ âge $»$. Pour cela, on utilise un tamis à maille ronde de $6 \mathrm{~mm}$ de diamètre permettant de séparer la population juvénile en deux lots :
— individus dont le grand diamètre de la coquille (D) est inférieure à $6 \mathrm{~mm}$ $(\mathrm{D}<6 \mathrm{~mm})$;
- individus dont le grand diamètre de la coquille (D) est supérieur ou égal à $6 \mathrm{~mm}(\mathrm{D} \geqslant 6 \mathrm{~mm})$.

A la fin de la seconde phase : «nursery $2^{\text {e }}$ âge ", on effectue un second tri avant de mettre les escargots dans le bâtiment «engraissement», ceci se faisant alors quand les individus sont âgés de 12 semaines.

\section{Mesures effectuées et échantillonnage}

Pour chacune des enceintes d'élevage, au début et à la fin de chaque phase "nursery $1^{\text {er }}$ âge » et "nursery $2^{\circ}$ âge », les biomasses d'escargots sont déterminées grâce à une balance «Mettler» au mg.

a) Phase «nursery $1^{\text {cr }}$ âge»

191 enceintes d'élevage sont utilisées et suivies pour cette phase. Les biomasses initiale et finale au bout de 6 semaines sont enregistrées afin de déterminer la production journalière, c'est-à-dire la quantité de matière vivante (animal + coquille) élaboré dans chaque enceinte par jour. De plus, tous les 14 jours, pour l'une de ces enceintes, choisie initialement au hasard comme «enceinte échantillon 》 (effectif initial : 2000 individus), chaque individu est pesé afin d'étudier sa vitesse de croissance, sa production journalière, l'évolution démographique de la population et la mortalité.

Enfin, afin de vérifier que la production des enceintes des escargots ne dépend pas de leur position respective sur les diverses étagères des portiques, nous déterminons et comparons les productions obtenues dans les 191 enceintes en les regroupant selon leur position.

b) Phase «nursery $2^{e}$ âge»

535 enceintes d'élevage sont suivies pour cette phase (321 enceintes pour $\mathrm{D} \geqslant 6 \mathrm{~mm}$ et 214 pour $\mathrm{D}<6 \mathrm{~mm}$ ) pour étudier la production journalière selon la taille des escargots. 
Comme pour la phase précédente, tous les 14 jours, pour l'une des 321 enceintes $\mathrm{D} \geqslant 6 \mathrm{~mm}$ et l'une des 214 enceintes $\mathrm{D}<6 \mathrm{~mm}$, choisies initialement au hasard comme "enceintes échantillons $2^{\mathbf{c}}$ âge », chaque individu est pesé pour étudier sa vitesse de croissance, sa production journalière, l'évolution démographique des populations retenues et les mortalités.

Enfin, on mène une expérience ayant pour but de voir si la production des enceintes varie selon la charge biotique et la taille des individus. Pour cela, on ieffectue le même protocole, mais avec une charge biotique de $50 \mathrm{~g} /$ enceinte $(\mathrm{N}=178$ enceintes).

\section{Résultats}

\section{Résultats globaux}

\section{A. Phase «nursery $I^{\text {er }}$ âge »}

Tout d'abord, on note que bien qu'il y ait eu une certaine mortalité des «jeunes 1 », la biomasse a largement augmenté, puisque la charge biotique atteint $1780 \mathrm{~g} / \mathrm{m}^{2}$ au sol ou près de $14 \mathrm{~kg}$ par $\mathrm{m}^{3}$ disponible, et que le poids moyen des escargots est passé de $28 \mathrm{mg}$ à $100 \mathrm{mg}$ en 6 semaines (tabl. 4).

\section{Tableau 4}

Principaux résultats concernant la population d'escargots «jeunes 1 》(Helix aspersa Müller) élevés dans l'enceinte échantillon, depuis leur éclosion jusqu'à l'âge de 6 semaines.

Main results on snail population «young $l \gg$ (Helix aspersa Müller) reared in the sample rearing-box, from hatching to 12 weeks of age.

\begin{tabular}{|c|c|c|}
\hline Paramètres & $\begin{array}{l}\text { Au temps: } \\
\mathrm{t}=1 \text { jour }\end{array}$ & $\begin{array}{l}\text { Au temps: } \\
t=42 \text { jours }\end{array}$ \\
\hline Effectif $\ldots \ldots \ldots \ldots \ldots \ldots \ldots \ldots \ldots$ & 2000 & 1432 \\
\hline Biomasse en $\mathrm{g} \ldots \ldots \ldots \ldots \ldots \ldots \ldots \ldots \ldots$ & 50,06 & 144,06 \\
\hline Charge biotique $/ \mathrm{m}^{2}$ au sol en $\mathrm{g} \ldots \ldots \ldots \ldots$ & 630 & 1780 \\
\hline Charge biotique $/ \mathrm{m}^{3}$ disponible en $\mathrm{kg} \ldots \ldots \ldots$ & 5,0 & 14,4 \\
\hline Poids moyen des escargots en $\mathrm{mg}(\overline{\mathrm{m}} \pm \sigma) \ldots \ldots$ & $28,0 \pm 8,0$ & $100,6 \pm 85,0$ \\
\hline $\begin{array}{l}\text { Valeur moyenne du grand diamètre } \mathrm{D} \text { de la co- } \\
\text { quille en } \mathrm{mm}(\overline{\mathrm{m}} \pm \sigma) \ldots \ldots \ldots \ldots \ldots \ldots \ldots\end{array}$ & $4,40 \pm 1,00$ & $7,80 \pm 5,80$ \\
\hline
\end{tabular}

\section{Evolution démographique de la population échantillon}

On note que pour les escargots nouveau-nés (1 jour), la population est homogène puisque 98 p. 100 des individus pèsent entre 8 et $50 \mathrm{mg}$ (fig. 3). Cette homogénéité, relativement stable au cours des 14 premiers jours, va très rapidement disparaître 
pour laisser place à une forte hétérogénéité de la population au bout de 6 semaines, les individus pesant entre 12 et $800 \mathrm{mg}$. Il devient alors nécessaire de pratiquer un tri des individus, sinon seuls les gros individus continuent de grossir tandis que les petits dépérissent et meurent (phénomène déjà observé antérieurement au cours d'expériences préliminaires).
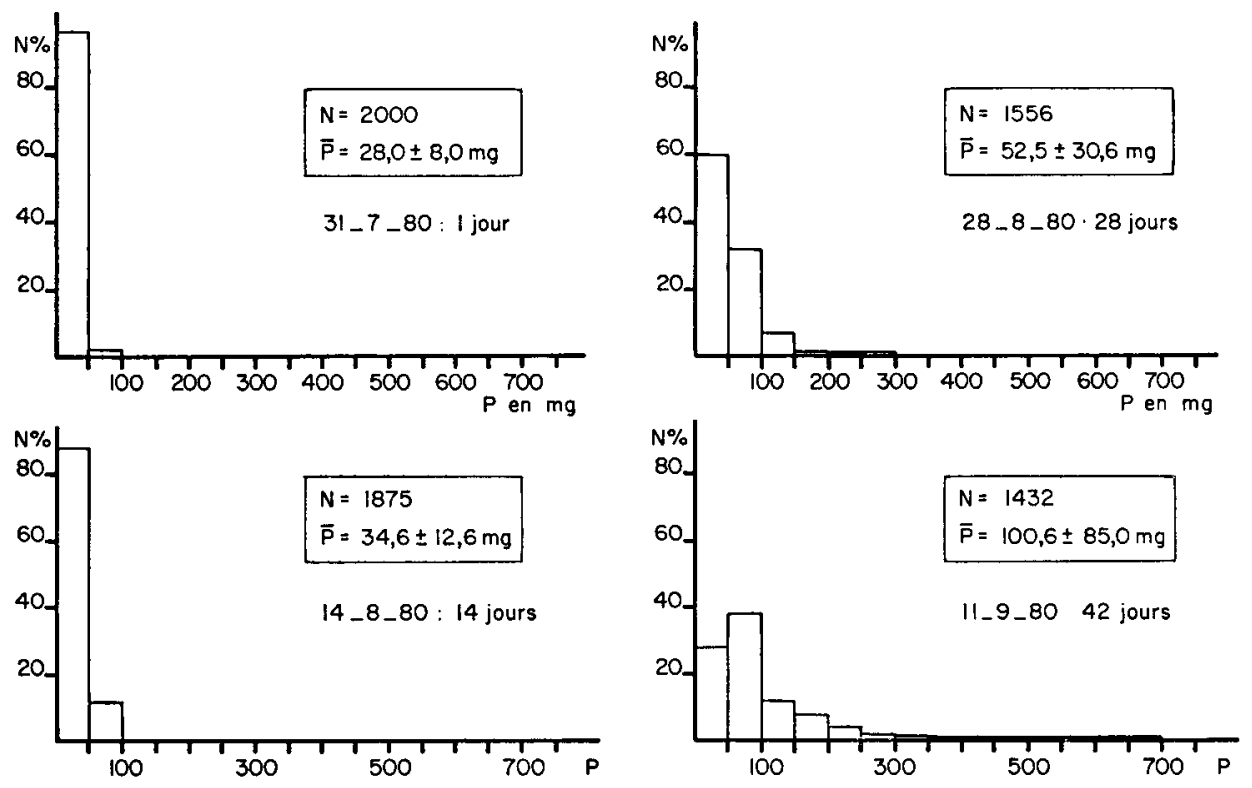

FIG. 3

Evolution démographique de la population échantillon de «jeunes 1 » d'Helix aspersa Müller, depuis l'éclosion jusqu'à l'âge de 6 semaines.

Demographic development of the population sample of Helix aspersa Müller "young 1 » from hatching to 6 weeks of age.

\section{Mortalité}

La mortalité relativement faible au bout de 14 jours (6 p. 100) va s'élever par la suite jusqu'au $28^{\circ}$ jour ( 22 p. 100), puis se stabiliser plus ou moins pour atteindre 28 p. 100 au bout de 6 semaines (fig. 4).

\section{Croissance pondérale}

La croissance pondérale des individus, relativement faible au cours des premiers 14 jours, va s'accélérer de plus en plus par la suite (fig. 5). Le poids moyen individuel va ainsi passer de $28 \mathrm{mg}$ à $100 \mathrm{mg}$ en 6 semaines.

En outre, on note que parallèlement à l'évolution du poids individuel, le coefficient de variation du poids va passer de 30 p. 100 à 85 p. 100 , ce qui indique une très grande hétérogénéité de la population d'escargots au bout de 6 semaines d'élevage (fig. 6). 


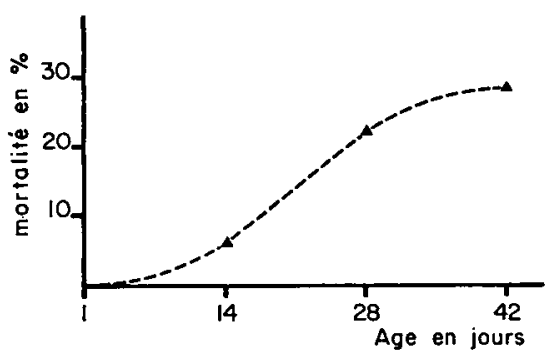

FIG. 4

Mortalité cumulée des individus "jeunes $I » d$ 'Helix aspersa Müller, depuis leur éclosion jusqu'à l'âge de 6 semaines.

Cumulated mortality of «young $I$ 》 Helix aspersa Müller individuals, from hatching to 6 weeks of age.

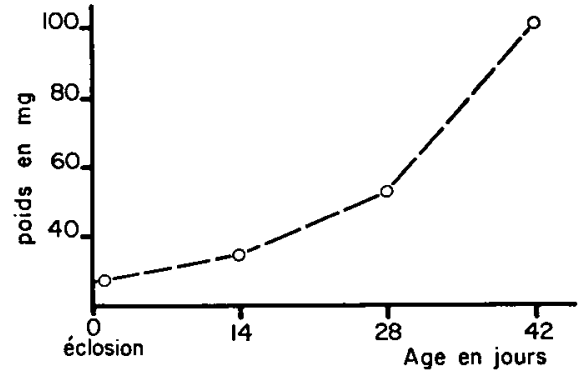

FIG. 5

Evolution du poids frais en fonction de l'âge chez les individus «jeunes 1 » d'Helix aspersa Müller $(N=2000)$.

Body weight development according to age in "young 1 » Helix aspersa Müller individuals $(N=2000)$.

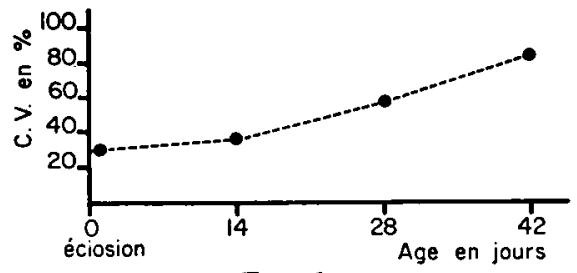

FIG. 6

Evolution du coefficient de variation du poids des individus "jeunes 1 » $d$ 'Helix aspersa Müller $(N=2000)$.

Body weight coefficient of variation in "young $l$ » Helix aspersa Müller individuals $(N=2000)$.

Si au sein de la population de «jeunes $1 »$, on compare la vitesse de croissance pondérale des individus les plus gros avec celle des individus les plus petits, en utilisant le taux de croissance pondérale par jour (r) établi par les auteurs britanniques CREese \& Underwood (1976) pour d'autres Mollusques Gastéropodes (2), on constate que la différence des taux de croissance est très significative (tabl. 5).

\section{Production}

a) Production individuelle

Au niveau de l'enceinte d'élevage «échantillon», il est possible de suivre l'évolution de la production journalière chez les individus "jeunes 1 ». On constate que la valeur de cette production triple à peu près tous les 14 jours pendant la phase «nursery $1^{\text {er }}$ âge » (tabl. 6).

(2) Selon Creese \& Underwood (1976), $r=\log \left(\frac{P_{2}}{P_{1}}\right) / t$ où $P_{1}$ est le poids au temps $t_{1}$, $P_{3}$ le poids au temps $t_{2}$, et $t$ le nombre de jours qui s'écoulent entre $t_{1}$ et $t_{2}$. 


\section{TABleau 5}

Variations de la croissance pondérale des individus « jeunes 1 » d'Helix aspersa Müller, selon la taille des escargots (durée 6 semaines).

Variations in the weight growth of «young 1 » individuals of Helix aspersa Müller in relation to snail size (during 6 weeks).

\begin{tabular}{c|c|c|c}
\hline Taille des individus & $\begin{array}{c}\text { Poids moyen } \\
\text { des' escargots } \\
\text { au temps } \\
\mathrm{t}=1 \text { jour }\end{array}$ & $\begin{array}{c}\text { Poids moyen } \\
\text { des escargots } \\
\text { au temps } \\
\mathrm{t}=42 \text { jours }\end{array}$ & $\begin{array}{c}\text { Taux } \\
\text { de croissance } \\
\text { pondérale } \\
\text { par jour (r) }\end{array}$ \\
\cline { 1 - 3 } Population entière $(\mathrm{N}=2000)$ & $28,0 \pm 0,2 \mathrm{mg}$ & $100,6 \pm 2,4 \mathrm{mg}$ & 0,030 \\
50 plus gros individus ....... & $53,1 \pm 0,8 \mathrm{mg}$ & $398,5 \pm 16,4 \mathrm{mg}$ & 0,055 \\
50 plus petits individus ...... & $10,6 \pm 0,3 \mathrm{mg}$ & $22,8 \pm 0,5 \mathrm{mg}$ & 0,018 \\
\hline
\end{tabular}

Moyenne \pm erreur-standard.

\section{Tableau 6}

Evolution de la production journalière chez les individus «jeunes $1 \gg$ d'Helix aspersa Müller, depuis leur naissance jusqu'à l'âge de 6 semaines.

Daily production in Helix aspersa Müller «young $1 \gg$ individuals from hatching to 6 weeks of age.

\begin{tabular}{|c|c|}
\hline Age des individus & Production journalière/individu \\
\hline De 1 jour à 14 jours ... & $0,47 \mathrm{mg} / \mathrm{j}$ \\
\hline De 14 jours à 28 jours & $1,30 \mathrm{mg} / \mathrm{j}$ \\
\hline De 28 jours à 42 jours & $3,41 \mathrm{mg} / \mathrm{j}$ \\
\hline
\end{tabular}

b) Production selon la position des enceintes d'élevage sur les portoirs

Si on essaie de voir si la production varie selon la place qu'occupe telle ou telle enceinte d'élevage sur les portiques, on constate qu'il n'y a aucune différence significative entre les divers niveaux occupés par les enceintes (tabl. 7). 


\section{TABleau 7}

Importance de la production chez les individus « jeunes 1 »d'Helix aspersa Müller selon la position des enceintes d'élevage sur les portoirs dans le bâtiment «nursery »

(durée 6 semaines).

Production performance in «young $1 》$ individuals of Helix aspersa Müller according to the place of rearing boxes on the porticos in the nursery building (during 6 weeks).

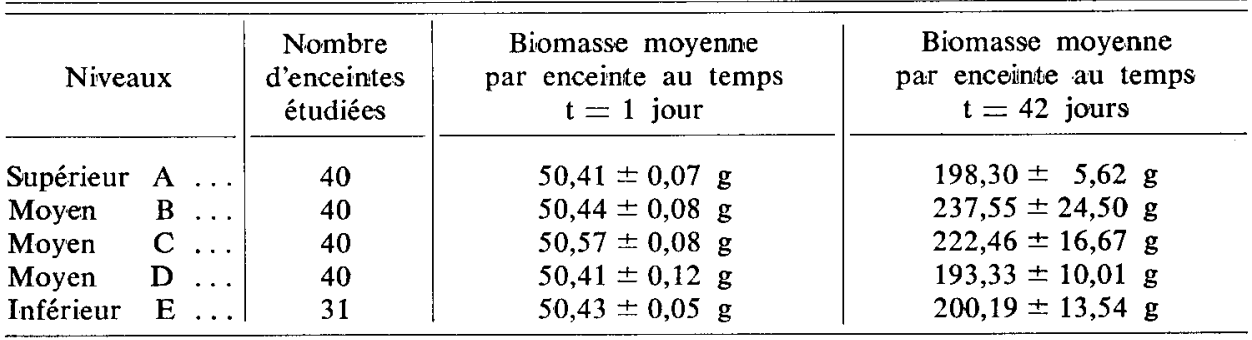

Moyenne \pm erreur-standard.

c) Variation de la production selon la date d'éclosion des escargots

Si l'on considère la valeur de la production chez les escargots «jeunes 1 » selon leur date d'éclosion, on note que les différences enregistrées entre les productions journalières des individus nés au cours des semaines successives sont significatives ou hautement significatives (test $t$ de Student) (tabl. 8).

TABLEAU 8

Variations de la production chez les individus «jeunes $I \gg d$ 'Helix aspersa Müller selon leur date d'éclosion (durée 6 semaines).

Production variations in «young $I$ » individuals of Helix aspersa Müller in relation to hatching date (during 6 weeks).

\begin{tabular}{|c|c|c|c|c|}
\hline Semaine d'éclosions & $\begin{array}{c}\text { Nombre } \\
\text { 1'enceintes } \\
\text { étudiées }\end{array}$ & $\begin{array}{c}\text { Biomasse moyenne } \\
\text { par enceinte } \\
\text { au temps } \\
\mathbf{t}=1 \text { jour }\end{array}$ & $\begin{array}{c}\text { Biomasse moyenne } \\
\text { par enceinte } \\
\text { au temps } \\
t=42 \text { jours }\end{array}$ & $\begin{array}{l}\text { Production } \\
\text { journalière } \\
\text { moyenne } \\
\text { par enceinte }\end{array}$ \\
\hline $1: 21 / 7-27 / 7$ & 7 & $50,39 \pm 0,02 \mathrm{~g}$ & $183,14 \pm 16,12 \mathrm{~g}$ & $3,16 \mathrm{~g} *$ \\
\hline $2: 28 / 7-3 / 8$ & 31 & $50,60 \pm 0,02 \mathrm{~g}$ & $142,36 \pm 6,93 \mathrm{~g}$ & $2,18 \mathrm{~g} * * *$ \\
\hline $3: 4 / 8-10 / 8$ & 40 & $50,42 \pm 0,01 \mathrm{~g}$ & $174,36 \pm 5,73 \mathrm{~g}$ & $2,95 \mathrm{~g} * * *$ \\
\hline $4: 11 / 8-17 / 8$ & 55 & $50,44 \pm 0,01 \mathrm{~g}$ & $182,19 \pm 6,19 \mathrm{~g}$ & $3,13 \mathrm{~g} * * *$ \\
\hline $5: 18 / 8-24 / 8$ & 26 & $50,44 \pm 0,01 \mathrm{~g}$ & $251,20 \pm 12,18 \mathrm{~g}$ & $4,98 \mathrm{~g}^{* * *}$ \\
\hline
\end{tabular}

Moyenne- \pm erreur-standard.

Différence significative entre 2 valeurs consécutives (test $t$ de Student).

$* * * \quad \mathrm{p}<0,001$.

$* * \mathrm{p}<0,01$.

$* \quad \mathrm{p}<0,05$. 
Il semble donc que plus les individus naissent tardivement au cours d'une même période de ponte, plus leur production est forte.

6) Bilan

A la suite de cette étude concernant la phase "nursery $1^{\text {er }}$ âge », il est possible d'en tirer un certain nombre de résultats intéressants pour le développement de l'héliciculture (tabl. 9).

\section{TaBleau 9}

Résultats concernant l'héliciculture pour la phase «nursery $1^{\text {er }}$ âge» en bâtiment chauffé à $20^{\circ} \mathrm{C}$ (durée 6 semaines, $N=200$ enceintes).

Heliciculture results for the period $《 N$ ursery $1 》$ in a heated building $\left(20^{\circ} \mathrm{C}\right)$

(duration 6 weeks, $N=200$ rearing-boxes).

\begin{tabular}{|c|c|c|}
\hline Paramètres & $t=1$ jour & $\mathrm{t}=42$ jours \\
\hline Biomasse par enceinte d'élevage en $g\left(\overline{\mathrm{m}} \pm \mathrm{S}_{\mathrm{m}}\right)$ & $\begin{array}{c}50,45 \pm 0,03 \\
(\text { C.V. }=0,8 \%)\end{array}$ & $\begin{array}{l}214,13 \pm 7,10 \\
(\mathrm{C} . \mathrm{V} .=46 \%)\end{array}$ \\
\hline
\end{tabular}

Production par enceinte

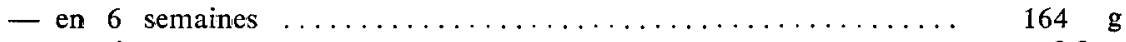

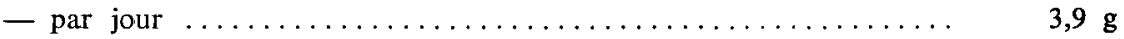

Production $/ \mathrm{kg}$ d'escargots « jeunes $1 » /$ jour $\ldots \ldots \ldots \ldots \ldots \ldots \ldots \ldots \ldots \ldots \ldots \ldots \ldots \ldots \ldots$

Taux moyen de production des escargots « jeunes 1 »

- en 6 semaines $\ldots \ldots \ldots \ldots \ldots \ldots \ldots \ldots \ldots \ldots \ldots \ldots \ldots \ldots \ldots \ldots \ldots \ldots \ldots \ldots, 2$

\section{Résultats globaux}

B. Phase «nursery $2^{e}$ âge»

Bien que la mortalité soit beaucoup plus importante dans les enceintes contenant des escargots dont le grand diamètre de la coquille (D) est inférieur à $6 \mathrm{~mm}$, quel que soit le type d'enceinte considéré, la biomasse, au bout de 6 semaines est sensiblement identique et la charge biotique est proche de $3 \mathrm{~kg} / \mathrm{m}^{2}$ au sol ou de $22 \mathrm{~kg} / \mathrm{m}^{3}$ disponible (tabl. 10).

\section{Evolution démographique des populations échantillons}

Si l'on considère l'enceinte contenant des escargots dont $\mathrm{D}$ est inférieur à $6 \mathrm{~mm}$, on constate que, bien qu'au départ la population soit relativement homogène ( 98 p. 100 des individus pesant entre 40 et $200 \mathrm{mg}$ ), elle devient très rapidement de plus en plus hétérogène au cours du temps (fig. 7). 


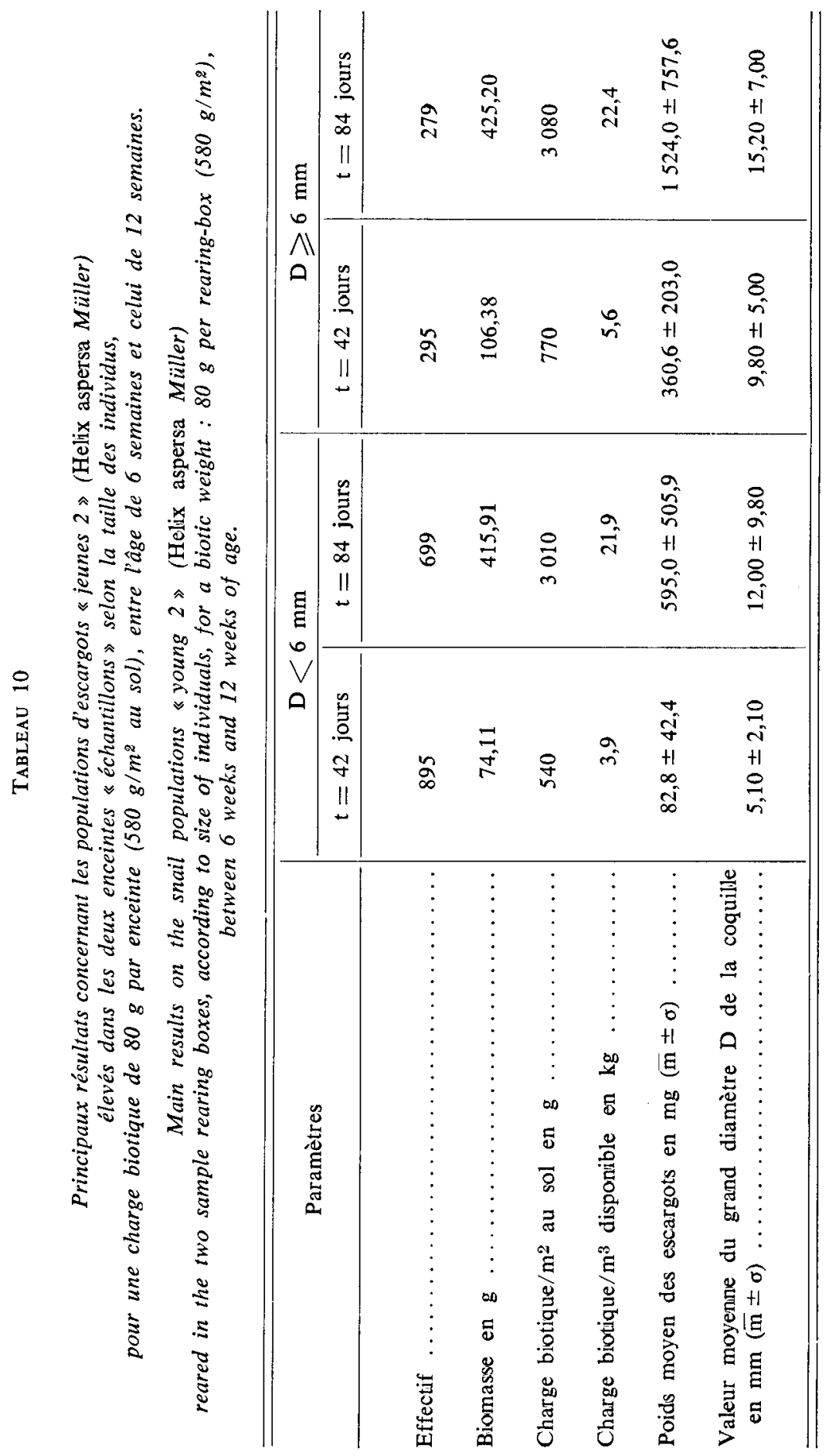



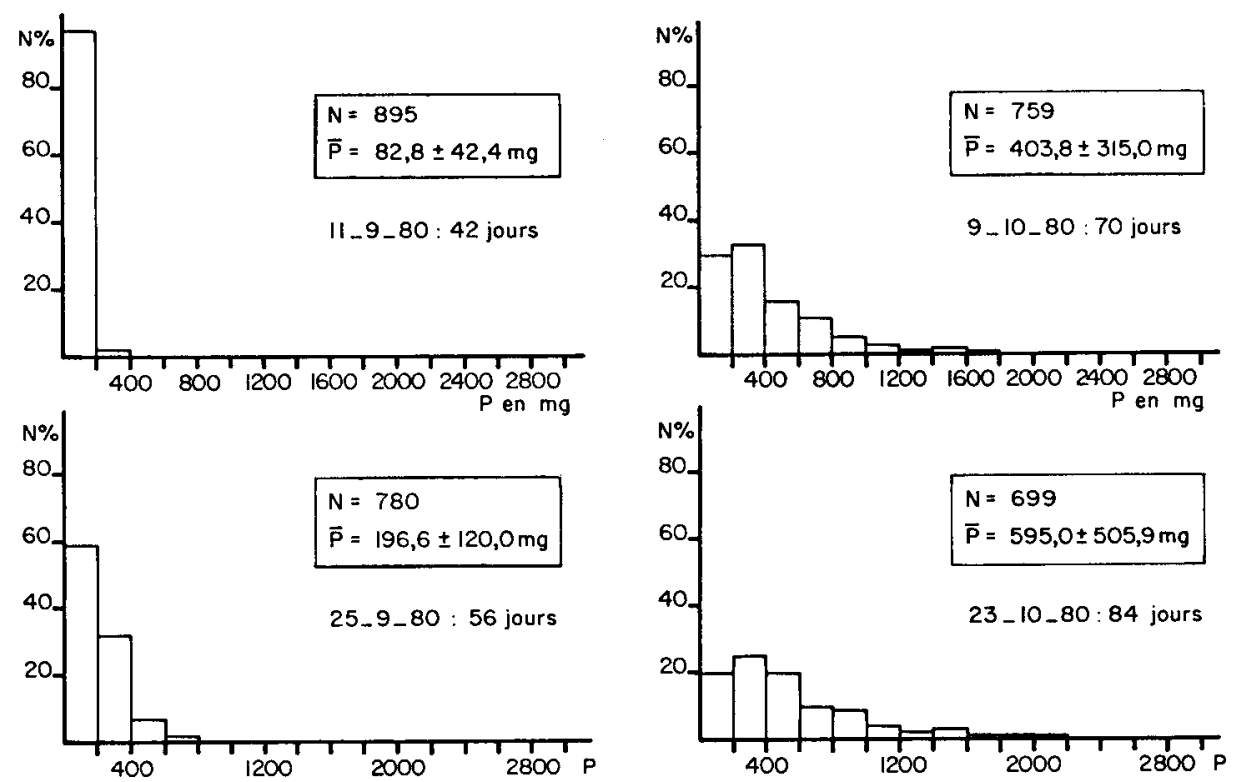

FIG. 7

Evolution démographique de la population échantillon de «jeunes 2 » les plus petits $(D<6 \mathrm{~mm})$ d'Helix aspersa Müller, entre l'âge de 6 semaines et celui de 12 semaines.

Demographic development of the smallest "young 2 »population $(D<6 \mathrm{~mm})$ of Helix aspersa Müller, between 6 weeks and 12 weeks of age.
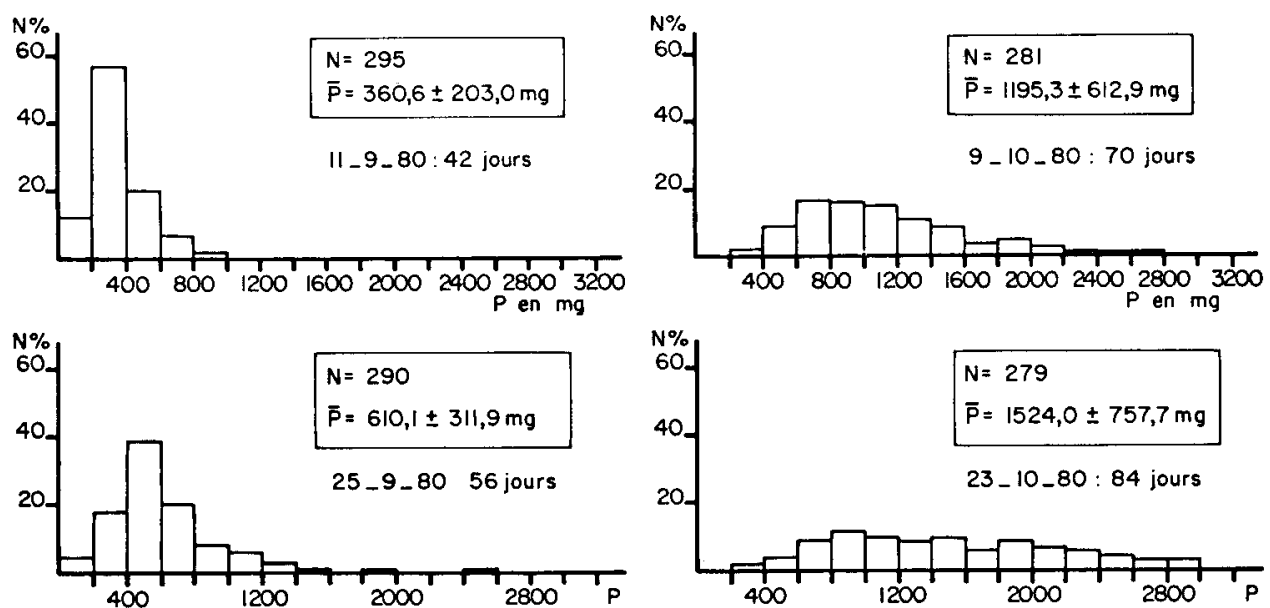

FIG. 8

Evolution démographique de la population échantillon de «jeunes 2 » les plus gros $(D \supseteq 6 \mathrm{~mm})$ d'Helix aspersa Müller, entre l'âge de 6 semaines et celui de 12 semaines.

Demographic development of the largest « young 2 »population $(D \supseteq 6 \mathrm{~mm})$ of Helix aspersa Müller, between 6 weeks and 12 weeks of age. 
De même, pour la population d'individus plus gros $(D \geqslant 6 \mathrm{~mm})$, on observe un phénomène identique (fig. 8).

\section{Mortalité}

La mortalité évolue lentement : 7 p. 100 au bout de 14 jours de nursery $2^{\circ}$ âge, 10 p. 100 au $28^{\circ}$ jour et 17 p. 100 à la fin de cette phase d'élevage (fig. 9). Cependant, la mortalité est différente selon la taille des individus mis dans les enceintes :

- 5 p. 100 pour les escargots dont $\mathrm{D} \geqslant 6 \mathrm{~mm}$,

- 21 p. 100 pour les escargots dont $\mathrm{D}<6 \mathrm{~mm}$.

Ainsi, les enceintes contenant les individus les plus petits $\mathrm{D}<6 \mathrm{~mm}$, présentent une mortalité 4 fois supérieure à celles contenant les escargots les plus gros $(D \geqslant 6 \mathrm{~mm})$.

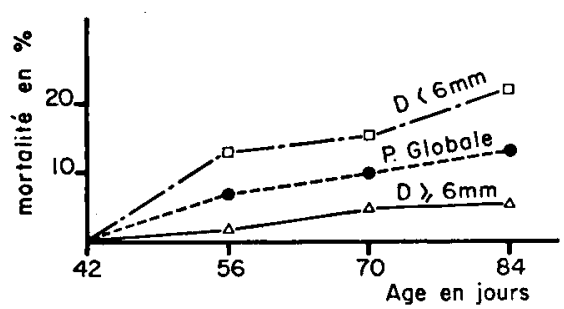

FIG. 9

Mortalité cumulée des individus " jeune 2 » $d$ 'Helix aspersa Müller entre l'âge de 6 semaines et celui de 12 sernaines.

Cumulated mortality of "young 2 » Helix aspersa Müller individuals between 6 weeks and 12 weeks of age.

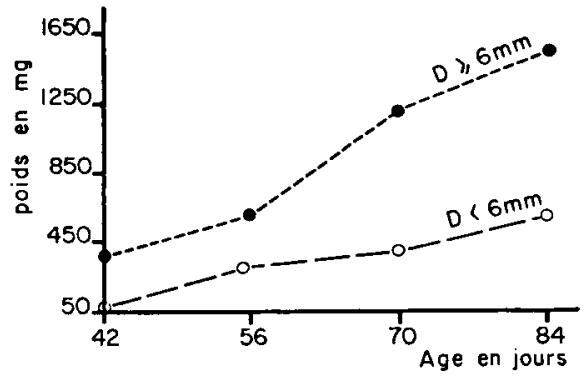

FIG. 10

Evolution du poids frais en fonction de l'âge et de la taille chez les individus «jeunes 2 » $d^{\prime}$ Helix aspersa Müller

$(D \supseteq 6 \mathrm{~mm}, N=295-D<6 \mathrm{~mm}$, $N=895$ ).

Body weight development according to age of «young 2 》

Helix aspersa Müller individuals $(D \supseteq 6 \mathrm{~mm}, N=295-D<6 \mathrm{~mm}$, $N=895$ ).

\section{Croissance pondérale et hétérogénéité de la population}

Quelle que soit l'enceinte considérée, on note qu'au début de la phase «nursery 2 », après le tri, la vitesse de croissance est la même. Cependant, par la suite, la croissance est beaucoup plus importante pour les gros individus que pour les petits (fig. 10).

$\mathrm{Si}$ on considère les coefficients de variation des poids individuels, on constate que le tri favorise une certaine stabilité de l'homogénéité des populations d'escargots les plus gros $(D \geqslant 6 \mathrm{~mm})$ (fig. 11$)$. 


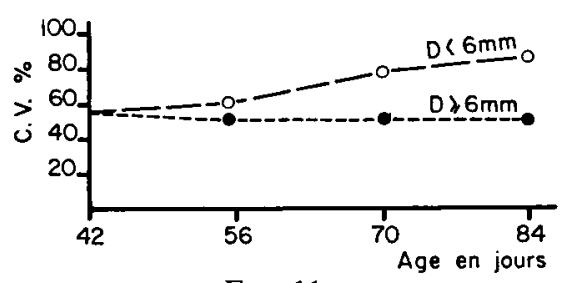

Fig. 11

Evolution du coefficient de variation du poids des individus "jeunes 2 » d'Helix aspersa Müller selon l'âge et la taille des escargots.

Body weight coefficient of variation in «young 2 »Helix aspersa Müller individuals according to snail age and size.

Par contre, pour les populations d'escargots les plus petits $(\mathrm{D}<6 \mathrm{~mm})$ après que le tri ait homogénéisé au début l'ensemble, très vite réapparaît une très grande hétérogénéité.

Si on compare la vitesse de croissance pondérale des individus les plus gros avec celle des individus les plus petits (tabl. 11), on enregistre les faits suivants :

- La vitesse de croissance est plus importante chez les escargots les plus petits (D $<6 \mathrm{~mm}$ ); il semble donc que le tri favorise le rattrapage du retard de croissance des individus les plus faibles.

- Au sein de la population de «jeunes 2 » ayant $\mathrm{D}<6 \mathrm{~mm}$, on note qu'il existe encore une différence de taux de croissance pondérale significative entre les individus les plus gros $(r=0,056)$ et les individus les plus petits $(r=0,029)$.

- Par contre, au sein de la population de « jeunes $2 »$ ayant $\mathrm{D} \geqslant 6 \mathrm{~mm}$, quelle que soit la taille des individus, le taux de croissance pondérale est toujours le même $(r=0,034)$.

Ainsi, le tri effectué à la fin de la phase «nursery 1 » favorise une meilleure homogénéité de la croissance des escargots les plus gros $(\mathrm{D} \geqslant 6 \mathrm{~mm})$, permet aux individus les plus petits $(\mathrm{D}<6 \mathrm{~mm})$ de rattraper leur retard de croissance, mais ne peut empêcher que les plus faibles des plus petits individus présentent une croissance médiocre.

\section{Production}

\section{a) Production individuelle}

On constate qu'elle est beaucoup plus importante que pendant la phase «nursery $1 \gg:$ quelle que soit la taille des individus, elle présente un maximum entre le $56^{\circ}$ et le $70^{\circ}$ jour, puis se ralentit (tabl. 12). Cependant, cette production est beaucoup plus élevée chez les gros individus que chez les petits.

b) Variation de la production selon la charge biotique et la taille des escargots

Si l'on réalise des enceintes d'élevage type «nursery $2^{\circ}$ âge », pendant 8 semaines les unes avec une charge biotique de $80 \mathrm{~g}$ par boîte et les autres avec une charge biotique de $50 \mathrm{~g}$ par enceinte, on constate que la production journalière par gramme d'escargot varie selon cette charge (tabl. 13). 


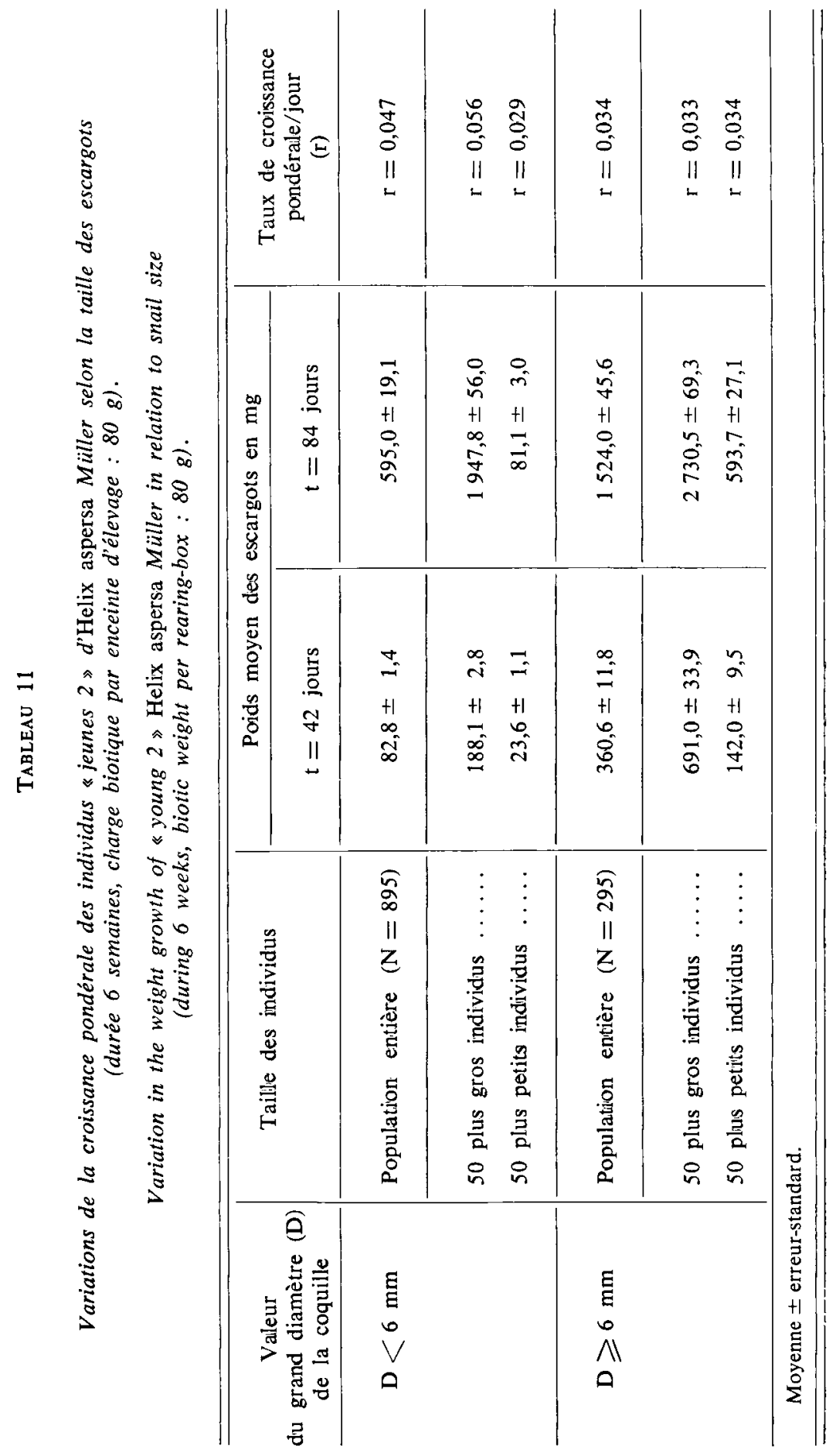




\section{TABleau 12}

Evolution de la production journalière chez les individus «jeunes $2 » d$ 'Helix aspersa Müller selon la taille des escargots, pour une charge biotique de $80 \mathrm{~g}$ par enceinte $\left(580 \mathrm{~g} / \mathrm{m}^{2}\right.$ au sol) entre l'âge de 6 semaines ct celui de 12 semaines.

Daily production in «young 2 » individuals of Helix aspersa Müller in relation to snail size, for a biotic weight : $80 \mathrm{~g}$ per rearing box $\left(580 \mathrm{~g} / \mathrm{m}^{2}\right)$ between 6 weeks and 12 weeks of age.

\begin{tabular}{|c|c|c|}
\hline \multirow{2}{*}{ Age des individus } & \multicolumn{2}{|c|}{ Production journalière/individu } \\
\hline & $\mathrm{D}<6 \mathrm{~mm}$ & $D \geqslant 6 \mathrm{~mm}$ \\
\hline De 42 jours à 56 jours & $8,13 \mathrm{mg} / \mathrm{j}$ & $17,86 \mathrm{mg} / \mathrm{j}$ \\
\hline De 56 jours à 70 jours & $14,80 \mathrm{mg} / \mathrm{j}$ & $41,80 \mathrm{mg} / \mathrm{j}$ \\
\hline De 70 jours à 84 jours & $13,60 \mathrm{mg} / \mathrm{j}$ & $23,52 \mathrm{mg} / \mathrm{j}$ \\
\hline
\end{tabular}

Grâce à l'utilisation du test $\mathrm{t}$ de Student, il est possible de montrer que :

- Quelle que soit la charge biotique considérée (50 g ou $80 \mathrm{~g}$ par boîte) ou la taille des individus ( $D \geqslant 6 \mathrm{~mm}$ ou $\mathrm{D}<6 \mathrm{~mm}$ ), le coefficient de variation de la production journalière par gramme d'escargot est identique (21 à 22 p. 100). biotique :

- Pour une même taille d'individus, la production varie selon la charge

- pour $\mathrm{D}<6 \mathrm{~mm}$, la production est plus importante pour une charge biotique de $50 \mathrm{~g} /$ enceinte (différence hautement significative);

- pour $D \geqslant 6 \mathrm{~mm}$, la production est plus élevée pour une charge biotique de $80 \mathrm{~g} /$ enceinte (différence hautement significative).

- Pour une même charge biotique par enceinte, il existe une différence hautement significative entre les boîtes contenant les plus petits individus $(D<6 \mathrm{~mm})$ et celles renfermant les plus gros escargots $(D \geqslant 6 \mathrm{~mm})$.

- La production journalière dépend de la charge biotique et de la taille des individus. Ainsi, on enregistre la même production pour une charge biotique de $50 \mathrm{~g}$ composée d'escargots ayant $\mathrm{D}<6 \mathrm{~mm}$ et pour une charge biotique de $80 \mathrm{~g}$ avec des individus dont $D \geqslant 6 \mathrm{~mm}$ d'autre part.

\section{Bilan}

Il est possible de noter un certain nombre de résultats intéressants pour la phase « nursery $2^{\mathrm{e}}$ âge * (tabl. 14). 


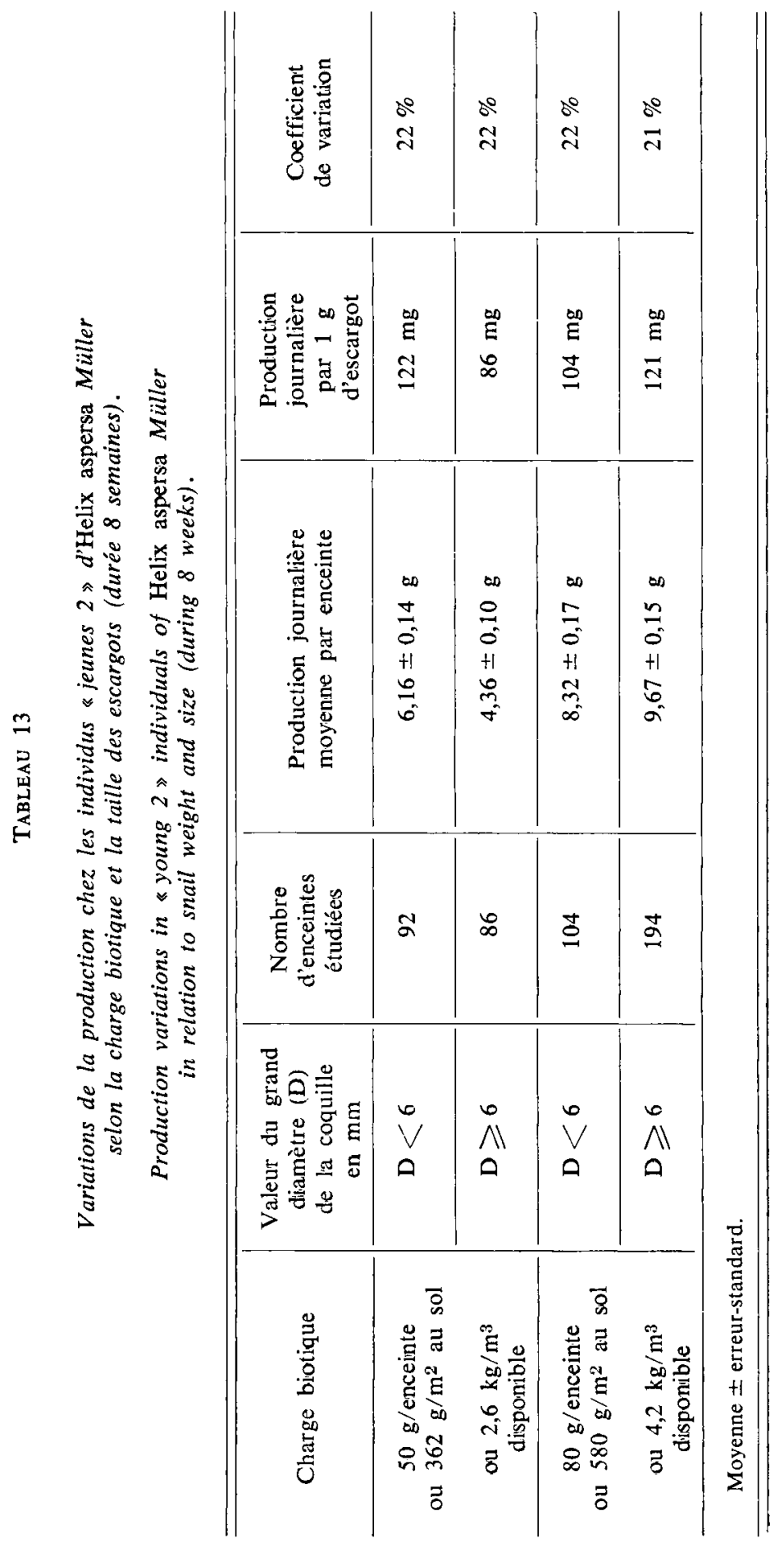




\section{TABLEAU 14}

Résultats concernant l'héliciculture pour la phase «nursery $2^{e}$ âge 》 en bâtiment chauffé à $20^{\circ} \mathrm{C}$ (durée 6 semaines, $N=535$ enceintes).

Heliciculture results for the period «nursery $2 》$ in a heated building $\left(20^{\circ} \mathrm{C}\right)$ (duration : 6 weeks, $N: 535$ rearing-boxes).

\begin{tabular}{|c|c|c|}
\hline Paramètres & $\mathrm{t}=42$ jours & $\mathrm{t}=84$ jours \\
\hline \multicolumn{3}{|l|}{$\begin{array}{l}\text { Biomasse par enceinte d'élevage en } g \\
\left(m \pm S_{m}\right) \text { : }\end{array}$} \\
\hline $\mathrm{D}<6 \mathrm{~mm} \ldots \ldots \ldots \ldots \ldots \ldots$ & $\begin{array}{c}80,23 \pm 0,02 \\
(\mathrm{C} . \mathrm{V} .=0,2 \%)\end{array}$ & $\begin{array}{l}545,96 \pm 11,18 \\
(\mathrm{C} . \mathrm{V} .=21 \%)\end{array}$ \\
\hline $\mathrm{D} \geqslant 6 \mathrm{~mm} \ldots \ldots \ldots \ldots \ldots \ldots$ & $\begin{array}{c}80,28 \pm 0,01 \\
(\text { C.V. }=0,2 \%)\end{array}$ & $\begin{array}{l}622,28 \pm 9,85 \\
(\mathrm{C} . \mathrm{V} .=22 \%)\end{array}$ \\
\hline
\end{tabular}

Production par enceinte :

- en 6 semaines :

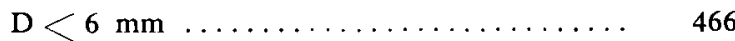

$\mathrm{D} \geqslant 6 \mathrm{~mm} \ldots \ldots \ldots \ldots \ldots \ldots \ldots \ldots \ldots, \quad 542 \quad \mathrm{~g}$

- par jour :

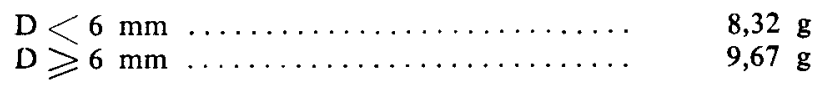

Taux moyen de production des escargots «jeunes 2 » en 6 semaines :

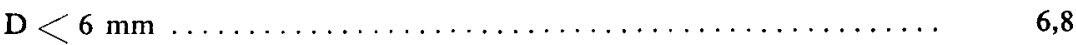

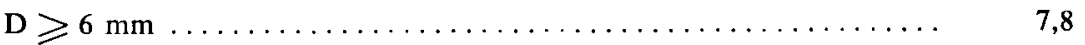

Importance relative de chaque type d'enceinte d'élevage d'escargots « jeunes 2 » (80 g d'escargots par enceinte) :

214 enceintes $\mathrm{D} \leqslant 6 \mathrm{~mm} \ldots \ldots \ldots \ldots$
321 enceintes $\mathrm{D} \geqslant 6 \mathrm{~mm} \ldots \ldots \ldots$

\section{Discussion et conclusion}

A la suite de cette étude originale, puisque jusqu'à présent, on ne possédait aucun résultat chiffré concernant l'évolution de la croissance des jeunes escargots Helix aspersa, à l'échelle «élevage», il est possible d'en tirer un certain nombre de remarques et de critiques.

- Tout d'abord, la mortalité relativement faible chez les jeunes individus «jeunes 1 au bout de 14 jours $\left(6 \mathrm{p}\right.$. 100) s'élève par la suite jusqu'au $28^{\circ}$ jour 
(22 p. 100), puis se stabilise pour atteindre 28 p. 100 à la fin de la $6^{\mathrm{C}}$ semaine, et enfin, est en moyenne de 40 p. 100 au bout de 12 semaines. Bien que cette valeur paraisse être importante, il ne faut pas oublier que dans la nature, 98 p. 100 des jeunes escargots meurent dans les 3 ou 4 mois qui suivent leur naissance, phénomène dû essentiellement à l'action de nombreux prédateurs et parasites (DaGUzaN, 1980). Par contre, cette mortalité observée en élevage est élevée par rapport à celle qu'on enregistre dans des conditions expérimentales, au laboratoire : environ 10 p. 100 en 3 mois (CHARrier, 1981). Cependant, nos résultats se rapportent à un élevage " grandeur nature » et les causes de la mortalité dans ce cas sont multiples : nettoyage moins fréquent ( 1 seule fois par semaine), mort accidentelle de plusieurs individus au cours de la manipulation des enceintes et des diverses pesées, etc. Il est bien évident qu'il convient, sur le plan technique, d'améliorer rapidement l'entretien de l'élevage afin de réduire au mieux la mortalité.

De plus, on note que chez les «jeunes $2 »$, la mortalité est plus importante pour les escargots de petite taille $(21$ p. 100$)$ que pour les plus gros $(5$ p. 100). Cela a toujours été observé, même en conditions expérimentales, au laboratoire. Il semble qu'il existe une compétition probablement de type alimentaire qui élimine de préférence les individus les plus faibles.

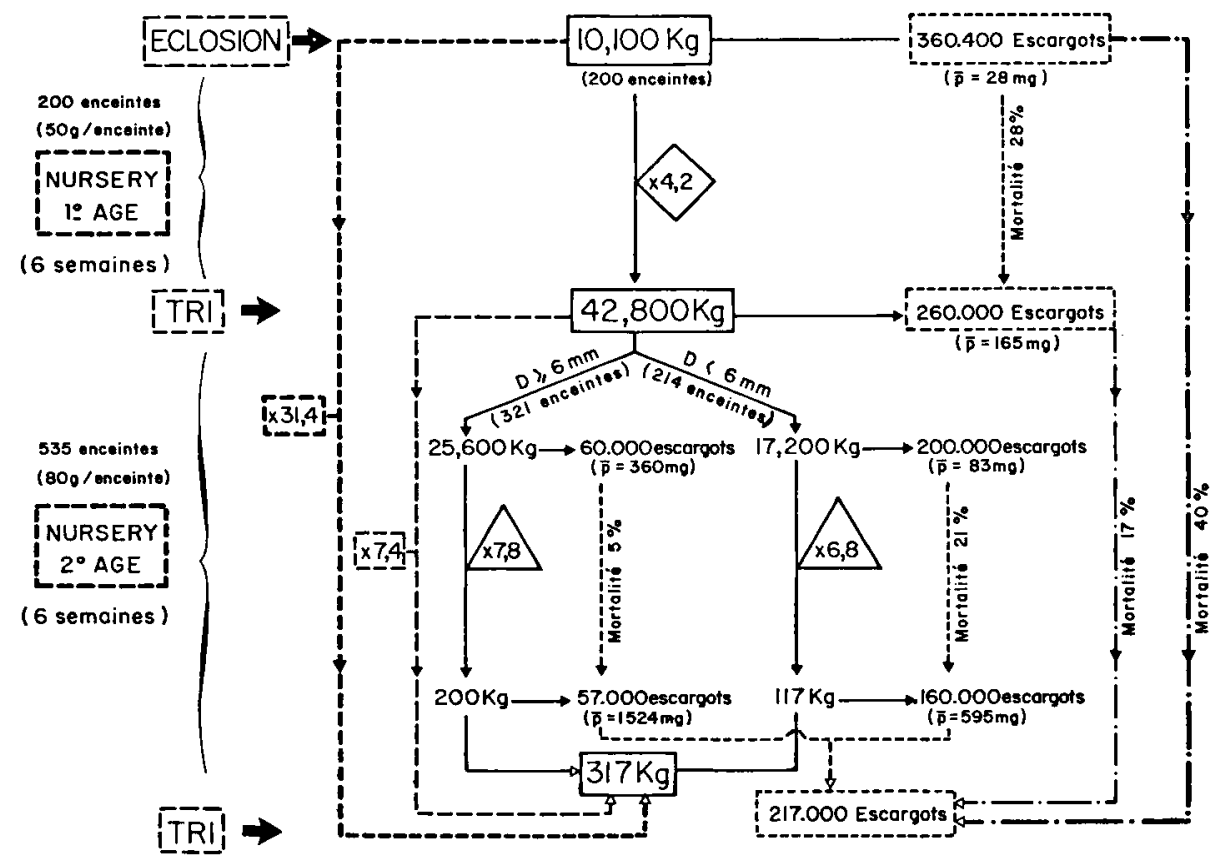

FIG. 12

Schéma récapitulatif de la phase "écloserie-nursery»

de l'élevage de l'escargot Petit-gris (Helix aspersa Mäller) réalisé en bâtiment chauffé à $20^{\circ} \mathrm{C}$

Recapitulative scheme of the «hatching-nursery» phase of Helix aspersa Müller reared in a heated building $\left(20^{\circ} \mathrm{C}\right)$. 
- L'homogénéité relative de la population des jeunes à l'éclosion, va très rapidement disparaître pour laisser place à une très forte hétérogénéité de taille des individus, phénomène caractéristique des Gastéropodes Pulmonés. Cependant, si on effectue un tri pour séparer les gros escargots des plus petits, on constate que cette opération favorise à la fois une certaine stabilité de l'homogénéité des populations d'escargots les plus gros, et le rattrapage du retard de croissance des individus les plus faibles. Par la suite, on note que pour les populations d'escargots les plus petits, très vite, réapparaît une très grande hétérogénéité de tailles. Il existe donc chez Helix aspersa une compétition alimentaire très importante qui favorise la croissance des individus les plus gros aux détriments des plus petits. Il est donc conseillé, au niveau de l'élevage, d'éliminer à la fin de la phase «nursery », à l'âge de 12 semaines, les individus les plus petits $(D<6 \mathrm{~mm})$ qui présentent une croissance très médiocre.

- L'élevage de l'escargot Petit-gris est possible, en bâtiment, en conditions thermo-hygrométriques contrôlées et en absence de terre, fait original qui évite les parasitoses dues surtout aux Nématodes et aux Acariens.

- Le fait que plus les individus naissent tardivement au cours d'une même période de ponte, et plus leur production est forte, semble montrer qu'il existe une certaine autorégulation naturelle qui ait pour but d'homogénéiser les populations de jeunes escargots.

- Il semble que la production varie selon la charge biotique. Il est donc nécessaire d'envisager des recherches afin de préciser la valeur de la charge biotique optimale selon l'âge des individus. Cependant, il conviendrait de ne pas négliger le facteur densité dont la valeur trop élevée pourrait entraîner un effet de masse et provoquer la perte de poids, voire la mort des jeunes escargots. De plus, selon OOSTERHOFF (1977), l'augmentation de la densité provoque une sécrétion importante de mucus qui, par «feed-back», inhiberait la locomotion et la consommation alimentaire, donc indirectement la croissance.

Enfin, ces résultats peuvent permettre aux héliciculteurs d'avoir des données de références pour leur élevage (fig. 12).

Accepté pour publication en mars 1982.

\section{Summary}

Contribution to production of «Petit-Gris» snails (Helix aspersa Müller)

II - Development of juveniles from hatching until 12 weeks of age under controlled housing and rearing conditions

The main results concerning the second phase of snail rearing, i.e. « development and growth of juveniles $\gg$ in heated houses $\left(20^{\circ} \mathrm{C}\right)$ with controlled hygrometry are given in this paper.

Several important results were found :

- the relatively low mortality after 14 days, reached 28 p. 100 on day 42 ; it was thereafter higher in small-sized $(21$ p. 100$)$ than in large-sized snails $(5$ p. 100), 
- the relative homogeneity of juveniles at hatching rapidly disappeared and a marked size heterogeneity of the snails was thereafter observed,

- the production varied according to the biotic load; production of individuals born the latest during one and the same hatching period seemed to be largest,

- if the big snails were separated from the smaller ones, a certain stability in the homogeneity of the large-sized snails, was observed as well as a recovery of the growth delay in the smaller sized individuals.

\section{R:́férences bibliographiques}

Abelcos M., 1944. Recherches cxpérimentalcs sur la croissance des Mollusques Arionidés. Bull. biol. Fr. Belg., 78, 215-256.

Charrier M., 1980. Contribution à la biologie et à l'écophysiologie de l'escargot «Petitgris », Helix aspersa Müller (gastéropode pulmoné stylommatophore). Thèse de doctorat $3^{e}$ cycle, Université de Rennes.

Charrier M., 1981. Contribution à l'étude de la croissance et de la consommation alimentaire de l'escargot Petit-gris Helix aspersa Müller (gastéropode pulmoné stylommatophore) élevé dans des conditions thermohygrométriques et photopériodiques contrôlées. Rapport $n^{\circ}$ C.G.D. 206/2 E.P.R., Bretagne, 91 pages.

Charrier M., Daguzan J., 1979. Etude de la croissance de l'escargot Petit-gris, Helix aspersa Müller (mollusque gastéropode pulmoné). Haliotis, 9, 15-18.

Chevallier H., 1969. Taxonomie et biologie des grands Arions de France (Pulmonata : Arionidae). Malacologia, 9, 73-78.

Chevallier H., 1974. Les grands Arions de France : taxonomie, biogéographie, écologie, polymorphisme, croissance et cycle biologique. Thèse de doctorat d'Université, Paris.

Creese R.G., Underwood A.J., 1976. Observations on the biology of the Trochid Gastropod Austrocochlea constricta (Lamarck) (Prosobranchia). I - Factors affecting shell-banding patter. J. exp. Biol. Ecol., 23, 211-228.

Daguzan J., 1980. Principales caractéristiques biologiques, écologiques et écophysiologiques de l'escargot Petit-gris Helix aspersa Müller. Doc. Technique I.T.A.V.I. sur "l'élevage de l'escargot $»$, Rennes, 8 pages.

Daguzan J., 1981. Contribution à l'élevage de l'escargot Petit-gris : Helix aspersa Muiller (mollusque gastéropode pulmoné, stylommatophore). I - Reproduction et éclosion des jeunes, en bâtiment et en conditions thermohygrométriques contrôlées. Ann. Zootech., 30, 249-272.

Dagczan J., 1982. Contribution à l'étude de la croissance et de la longévité de Elona quimperiana (de Ferussac) (Gastéropode Pulmoné stylommatophore) vivant en Bretagne occidentale. Malacologia, 22, 385-394.

Herzberg A., Herzberg F., 1960. The effect of cold on the growth of Helix aspersa. J. exp. Zool., 145, 191-196.

LAmotte M., 1951. Recherches sur la structure génétique des populations naturelles de Cepaea nemoralis. Bull. biol. Fr., 35, 1-239.

Lazaridou-Dimitriadou M., 1978. Contribution à l'écophysiologie d'un gastéropode pulmoné dunicole Euparypha pisana (Müller) du littoral armoricain. Thèse de doctorat de $3^{\text {e }}$ cycle, Université de Rennes.

Lazaridou-Dimitriadou M., Daguzan J,, 1981. Etude de l'effet du «groupement» des individus chez Theba pisana (Mollusque gastéropode Pulmoré stylommatophore). Malacologia, 20, 195-204.

Oosterhoff L.M., 1977. Variation in growth rate as an ecological factor in the land snail Cepaea nemoralis L. Neth. J. Zool., 27, 1-132.

Pollard E., 1973. Growth classes in the adult Roman snail (Helix pomatia L.). Oecologia, 12, 209-212. 
Pollard E., 1975. Aspects of the ecology of Helix pomatia L. J. anim. Ecol., 44, 305-329. Pollard E., Cooke A.S., Welch J.M., 1977. The use of shell features in age determination of juvenile and adult Roman snails Helix pomatia. J. Zool., 183, 269-279.

PotTs D.C., 1972. Population ecology of Helix pomatia and the nature of selection in favorable and unfavorable environments. PhD Thesis, University of California, SantaBarbara.

STERN G., 1968. Recherches sur le bilan énergétique de la limace Arion rufus (L.) en période de croissance. Doctorat de $3^{\mathrm{e}}$ cycle, Université de Paris.

Williamson P., 1976. Size-weight relationships and field growth rates of the landsnail Cepaea nemoralis L. J. anim. Ecol., 45, 875-885.

Wolda H., 1963. Natural populations of the polymorphic landsnail Cepaea nemoralis L. Arch. néerl. Zool., 15, 381-471.

WoldA H., 1971. Variations in the growth rate in the landsnail Cepaea nemoralis L. Res. Popul. Ecol., 12, 185-204.

Wolda H., 1972. Ecology of some experimental populations of the landsnail Cepaea nemoralis L. I : Adult numbers and adult mortality. Neth. J. Zool., 22, 428-455.

Wolda H., 1973. Changes in shell size in some experimental populations of the landsnail Cepaea nemoralis L. Argamon Isr. J. Malacol., 3, 63-71. 